\title{
Avances en los estudios migratorios. Nuevos enfoques, nuevos instrumentos en el estudio de la movilidad internacional.
}

PRESENTACIÓN

\author{
Ana López-Sala \\ Consejo Superior de Investigaciones Científicas (CSIC)
}

Los estudios migratorios han experimentado un gran desarrollo a lo largo de las últimas dos décadas. Este desarrollo, que ha llevado a este campo a contar con una presencia específica y distintiva en las ciencias sociales, no sólo ha supuesto un incremento del volumen en la producción científica en torno a la movilidad humana, sino que se ha acompañado de procesos de institucionalización e internacionalización. Desde el comienzo de siglo se han consolidado los programas de formación e investigación no sólo en Europa y Norteamérica, sino también en Latinoamérica y Asia. También ha aumentado significativamente el número de revistas académicas especializadas, así como su calidad y alcance y las redes de investigación internacionales multidisciplinares. Aunque la presencia de especialistas procedentes del mundo académico anglosajón sigue siendo mayoritaria, en la última década ha aumentado también la de investigadores procedentes de otras geografías. Esta «mayoría de edad» de los estudios migratorios (Pisarevskaya, Levy, Scholten y Jansen, 2019) ha corrido paralela a la centralidad que la movilidad humana ha llegado a adquirir en la agenda política y en las dinámicas sociales y económicas internacionales. Se trata por tanto hoy de un área bien establecida, con una clara vocación multidisciplinar, crecientemente multisituada y en donde abundan los estudios comparados y la diversidad en los niveles de análisis.

Sin embargo, hasta fechas recientes, los avances en la teoría migratoria han sido más modestos. Ello se debe, han sostenido diversos autores, a la continuidad de la fragmentación disciplinar en este panorama académico (Bretell y Hollifield, 2008), así como a la dificultad para acuñar teorías globales y de amplio alcance en un contexto de creciente complejidad y de diversificación de los tipos de flujos, incluidos los de refugiados (Arango, 2004; Van Hear, 2010; King, 2012; Bloch, 2020). A pesar de ello, desde el cambio de siglo, se ha observado una transformación teórica relevante que surge, inicialmente, de la introducción de dos nuevos paradigmas conceptuales que han dominado la investigación en este campo: el transnacionalismo y el denominado «giro de la(s) movilidad(es)» (Urry, 2007; Glick Schiller, Basch y Blanc-Szanton, 1992). Adicionalmente, du- 
rante la última década, los estudios migratorios han sido un campo desde el que releer a algunos teóricos seminales de las ciencias sociales del siglo XX, entre otros, aunque la lista es larga, a Bourdieu, Foucault, Deleuze o Said.

Más recientemente, los caminos teóricos más transitados han dado paso a nuevas interpretaciones y enfoques que no sólo descansan en las aproximaciones clásicas, sino que amplían, avanzan y desafían a estas. La creciente complejidad y transversalidad de la movilidad humana y su impacto en múltiples procesos sociales parece haber impulsado esta ampliación epistemológica en donde también ha sido seminal un creciente diálogo interdisciplinar. En este nuevo panorama de los estudios migratorios han surgido nuevas aproximaciones críticas y conceptos teóricos emergentes.

Los estudios migratorios se han convertido, asimismo, en un campo donde se han ensayado y aplicado nuevos enfoques, herramientas e instrumentos metodológicos, algunos pioneros en las ciencias sociales, otros activados por las posibilidades que ofrecen los avances tecnológicos, que han mostrado la potencialidad que ofrecen otras formas de recogida y análisis de información que pretenden adaptarse de forma más ajustada a procesos y dinámicas cambiantes, en muchos casos de difícil cuantificación y medición. El ensayo de nuevas formas de recoger, producir y analizar datos, junto con las aportaciones conceptuales y teóricas de los últimos años muestran un panorama académico sometido a grandes cambios que promete, sin embargo, amplios avances.

El objetivo de este número monográfico es presentar y profundizar en algunos de estos nuevos enfoques teóricos e instrumentos empíricos en el campo de los estudios migratorios, subrayando las aportaciones que ofrecen y su influencia en las modulaciones emergentes de este campo de estudio de las ciencias sociales. La amplitud de estos nuevos enfoques y de las metodologías e instrumentos emergentes superan las posibilidades de un monográfico, que en este caso no pretende ser exhaustivo, pero que sí busca iniciar un camino de revisión y de discusión necesario en los estudios migratorios en nuestro país. Por ello se han seleccionado aproximaciones que han despertando un gran interés en el debate académico internacional, pero que tienen una escasa presencia en los estudios migratorios en España. Estas aproximaciones, de nuevo cuño, algunas ya con cierto desarrollo en el ámbito internacional, ofrecen un buen panorama de los retos, las nuevas direcciones y el porvenir de esta esfera de estudio.

Las aproximaciones teóricas que recorren la primera parte del monográfico (Ecología-Mundo, Industria de la Migración, Autonomía de la Migración y Actos de Ciudadanía) comparten algunos elementos. Se trata de enfoques que se alejan en cierta medida de la impronta de los estudios sobre el transnacionalismo, interdisciplinares y crecientemente influyentes, a pesar de que surgieron en los márgenes. Además en muchos casos resultan de un diálogo entre el conocimiento producido por la academia, el activismo y los propios migrantes. En todos los casos, se trata de aproximaciones todavía en fase de consolidación, sometidas a ampliación, revisión y contraste empírico. La segunda parte del monográfico tiene la intención de profundizar en algunas de las «nuevas formas» de investigar las migraciones, incluyendo algunas metodologías con mayor tra- 
yectoria en los estudios españoles (análisis de redes sociales) y otras que están dando sus primeros pasos (estudios cualitativos longitudinales y big data y redes sociales). Quedan otras muchas posibilidades (teóricas, conceptuales y metodológicas) en el tintero, que deberían ser objeto de nuevas compilaciones.

«Ecología-Mundo, un nuevo paradigma para el estudio de las migraciones internacionales» se centra en este novedoso enfoque, que surge en el campo de las Relaciones Internacionales y que se ha incorporado a los estudios migratorios recientemente. El primer objetivo del artículo es describir sus antecedentes y las fuentes teóricas en las que descansa. A lo largo de las primeras secciones, se presenta de forma muy sucinta la evolución de la escuela estructuralista de Relaciones Internacionales, así como la influencia de la teoría del sistema-mundo de Wallerstein destacando algunos conceptos fundamentales que son posteriormente recogidos por esta teoría. Acuñada por Jason W. Moore, parte de la premisa, indican los autores, de que la naturaleza es un elemento determinante para comprender la evolución del sistema-mundo y que el capitalismo es un proyecto socio-ecológico que permite la acumulación, movilizando y apropiándose de las naturalezas humanas (trabajadores) y extrahumanas (naturaleza). Este proceso de apropiación es analizado en la siguiente sección del artículo a través de la introducción de dos conceptos clave: "los cuatro factores baratos" y la "frontera de producción". La segunda parte del artículo de Yoan Molinero y Gennaro Avallone se detiene tanto en el encaje de la movilidad humana en esta teoría como en el análisis de las migraciones a través de las lentes de la Ecología-Mundo. Usando como referencia los conceptos mencionados anteriormente, los autores analizan los vínculos entre fronteras de producción y desplazamientos de población, emigración y relaciones poscoloniales y factores baratos y producción de mano de obra migrante. Aunque concluyen que las investigaciones realizadas desde la Ecología-Mundo se han centrado especialmente en el trabajo migrante agrícola, así como en las migraciones ambientales, caracterizando ambos procesos como dinámicas constitutivas de la Ecología-Mundo, en su opinión se trata de un enfoque emergente, pero con grandes potencialidades para realizar aportaciones en los estudios migratorios más allá de las líneas temáticas abordadas hasta la fecha.

El artículo "Las Industrias de la Migración. ¿Un Nuevo Enfoque en el Análisis de la Movilidad Internacional?" se detiene en el protagonismo que este concepto ha adquirido en el léxico académico de los estudios sobre la movilidad internacional recientemente. El auge de este enfoque se enmarca en la incorporación analítica de los actores privados en el estudio de los determinantes y la modulación de las migraciones. El alejamiento del determinismo estatal que ha imperado en los estudios sobre la movilidad humana y su concreción a través del enfoque de la industria de la migración ha ofrecido nuevas perspectivas para entender la articulación contemporánea de las interacciones entre los Estados, el sector privado y los propios migrantes. Adicionalmente, su desarrollo como prisma analítico parte de la consideración de que las dinámicas y la gestión de los flujos migratorios internacionales no pueden entenderse adecuadamente sin tomar en consideración tanto los procesos de mercantilización y generación de beneficios que cristalizan en torno a ellos, como las prácticas neoliberales que 
han alcanzado a muchas de las esferas de la gestión migratoria. Es por tanto en la literatura de intersección sobre las nuevas formas que ha adoptado la regulación migratoria y los mecanismos de intermediación y privatización donde este enfoque ha recibido mayor atención. Se trata, sin embargo, sostiene la autora, de una perspectiva todavía en vías de consolidación, en donde siguen abiertas amplias controversias, no sólo en torno a la propia definición del concepto, sino en torno a su alcance, contorno y actores. Muchos de los contenidos, consensos y disensos que han aparecido en estos debates son analizados en este artículo, cuyo objetivo es presentar y analizar la conformación de este enfoque, los dilemas que han emergido en el seno de esta literatura, así como reflexionar críticamente sobre sus principales contribuciones en el campo de los estudios migratorios. En este sentido, y enmarcado en el presente monográfico, el artículo pretende realizar una aportación que transcienda la mera descripción de esta perspectiva para interrogarse, entre otros, sobre sus peculiaridades como enfoque, su diálogo con otras orientaciones influyentes o, de forma muy sucinta, sus vínculos con otras teorías.

El artículo de Maribel Casas-Cortés y Sebastian Cobarrubias realiza una introducción de la teoría de la Autonomía de la Migración (AoM). Esta teoría ha tenido una importante repercusión en los estudios migratorios en la última década, especialmente en los enfoques en los que confluye el análisis de la movilidad internacional y los estudios críticos fronterizos. Si bien surge en círculos académicos y de activistas muy vinculados a las "luchas migrantes", que le dotaron de un carácter de teoría o aproximación "en los márgenes", hoy se encuentra entre las nuevas teorías más influyentes, como muestra el interés y el eco que despiertan las publicaciones de algunos de sus autores más destacados. La primera parte del artículo se detiene en el concepto de autonomía y explora uno de sus supuestos más relevantes por su impacto epistemológico y metodológico: la comprensión de la migración como "movimiento social". En la segunda parte se realiza una genealogía de esta teoría dando a conocer los múltiples contextos en los que surge y su difusión a través de autores y disciplinas que han devenido en lo que los autores etiquetan como una "multiplicación de versiones" de la AoM. A pesar de estas diferencias los teóricos que se inscriben en esta aproximación comparten no solo la idea de la centralidad del concepto de "fuga", sino de la migración como un motor de transformaciones políticas (fronterizas, laborales y de ciudadanía, entre otras). El artículo concluye con un repaso de las principales críticas que ha recibido, especialmente las que destacan el proceso de "romantización" de la migración y las que han apelado a la reducción analítica de las singularidades y de las diversas experiencias de movilidad bajo la categoría genérica y única de "migrante". Los autores cierran su artículo subrayando lo que en su opinión son dos de los elementos distintivos y quizás más idiosincráticos de esta aproximación: a) el entendimiento de la migración como una fuerza dinámica que transforma procesos políticos, pero también económicos y culturales; b) su alejamiento, como paradigma o enfoque, de las preguntas teóricas clásicas de los estudios migratorios (las causas y los determinantes de la migración), para detenerse en entender cómo la migración condiciona las sociedades actuales. 
En el artículo "Cuando el derecho a fugarse subvierte el neo-orientalismo" Iker Barbero emplea la teoría de los Actos de Ciudadanía para analizar los procesos de resistencia de varios grupos de inmigrantes surasiáticos que fueron confinados durante años en Centros de Estancia Temporal para Inmigrantes en Ceuta y Melilla. Para evitar ser deportados, optaron por fugarse de los CETI sin ser conscientes de que su lucha derivaría en una serie de acciones y movimientos de solidaridad por el derecho a ser transferidos al continente europeo e impedir su deportación. Empleando diferentes fuentes de información, el artículo emplea la técnica de "escribir un acto de ciudadanía" de Engin Isin para capturar actos que quedarían ocultos bajo teorías dominantes de ciudadanía formal. La primera parte del artículo enmarca esta acción de fuga (y de lucha de migrantes) en la teoría de los Actos de Ciudadanía, una de las aproximaciones teóricas de mayor alcance y repercusión en los estudios de ciudadanía e inmigración desde principios de siglo, especialmente en la academia anglosajona. Este encaje se detiene en estas luchas migrantes en el seno de la conformación de los regímenes fronterizos (a través del concepto de neo-orientalismo) y a través de la escritura de estos actos, que centran los siguientes apartados del artículo, pretende indagar en la redefinición de la subjetividad política y legal de estos migrantes. En la última parte del artículo, se emplean analíticamente estos actos de ciudadanía para aportar algunos hallazgos, como conclusiones, que sirven para enriquecer y profundizar en la propia teoría. En opinión del autor, la idea de la ciudadanía como un estatus legal-formal estático no ofrece un marco suficiente para analizar las luchas de los migrantes, porque son precisamente estas luchas las que expanden los márgenes de la ciudadanía y la hacen dinámica; y, en segundo lugar, porque estos repertorios de contestación ocurren en un nivel alternativo al tradicionalmente vinculado al Estado-nación.

El artículo "El proceso intencional/decisorio migratorio desde una aproximación cualitativa longitudinal" se centra en una de las cuestiones que más atención ha despertado en los estudios migratorios: la adopción de las decisiones migratorias. Partiendo de la importancia de la dimensión temporal en la comprensión del fenómeno intencional y, por tanto, de la necesidad de adoptar una visión dinámica, el artículo de Renata Hosnedlová emplea una perspectiva cualitativa longitudinal. Esta perspectiva ha sido considerada, como se indica, la más idónea para explorar los procesos causales de la temporalidad de los sucesos, pero a pesar de esta idoneidad no ha recibido mucha atención en el campo de la migración intencional. Deteniéndose en un estudio de caso, el de la comunidad ucraniana en la Comunidad de Madrid, y empleando este tipo de metodología, el artículo analiza cómo se construyen, modifican y mantienen las intenciones/ decisiones migratorias a lo largo del tiempo en un contexto de incertidumbre. Estas decisiones migratorias se concretan analíticamente en el estudio del retorno, el asentamiento y la re-emigración. El propósito del artículo es evaluar la capacidad del enfoque longitudinal cualitativo para comprender el proceso del proyecto migratorio y para discernir la relación causal entre él, los factores macroestructurales y el entorno relacional de los inmigrantes ucranianos. El artículo no sólo presenta las aportaciones de la investigación, sino que se centra también 
en las dificultades y los retos conceptuales, metodológicos y analíticos de este enfoque para el estudio del proceso intencional / decisorio en las migraciones internacionales. La parte final del artículo concluye presentando las ventajas del enfoque longitudinal cualitativo, frente otras estrategias metodológicas, para el estudio de las decisiones migratorias, así como las limitaciones que presenta y concluye con algunas reflexiones finales, de enorme interés práctico, sobre cómo planificar y llevar a cabo un estudio con este enfoque.

A partir de una revisión de la bibliografía existente en este campo, enriquecida con la experiencia desarrollada en los últimos años por las autoras, Estrella Gualda y Carolina Rebollo analizan qué tipo de investigaciones se están realizando internacionalmente en el área de confluencia entre big data y estudios migratorios y, en particular, entre Twitter y estudios migratorios. El objetivo de su artículo es conocer las aportaciones metodológicas que para las Ciencias Sociales supone abordar estos campos de estudio, así como plantear horizontes de interés e identificar aspectos de carácter técnico que merece la pena resolver para el desarrollo de investigaciones basadas en la información difundida a través de diversas plataformas de redes sociales. En la primera parte de "Big data y Twitter para el estudio de procesos migratorios: Métodos, técnicas de investigación y software" las autoras sitúan la evolución internacional de la bibliografía publicada para dimensionar esta línea de investigación a partir de la explotación de las bases de datos de ProQuest, Scopus y WoS. Con ello obtienen un mapeo muy completo de la investigación actualmente publicada. El análisis arroja que este tipo de estudios son aún escasos, aunque crecientes, con una pauta de crecimiento sostenida en el último lustro. Los detallados resultados de la explotación presentados en la segunda parte del artículo se centran en las fuentes de información empleadas, los métodos de recolección y extracción de datos, de procesamiento (incluyendo estrategias de filtrado y de codificación y categorización) y el análisis de datos (métodos y técnicas de visualización) de los artículos analizados. Basándose en su trabajo y en el de otros investigadores que se han adentrado en esta línea de confluencia entre big data y migraciones, las autoras valoran muy positivamente las aportaciones y oportunidades que este tipo de investigaciones pueden introducir en el campo de los estudios migratorios. Si bien, matizan, ello debe suponer fortalecer las estrategias de triangulación y combinación metodológica, ante la variedad de métodos, técnicas, herramientas y fuentes de análisis que pueden, potencialmente, ser empleadas.

El artículo de Verónica de Miguel, que cierra el monográfico, se detiene en la utilización de la metodología de análisis de redes sociales (ARS) en el campo de los estudios migratorios. Tras una introducción muy breve y de corte más teórico, que recorre los conceptos de redes, cadenas migratorias y capital social como fundamento de los estudios en los que se aplica esta metodología, el artículo profundiza en los análisis sobre las redes sociocéntricas y las redes egocéntricas, incluyendo los mecanismos de recogida de datos y las técnicas de análisis más habituales. A través de sus apartados se va describiendo el uso de esta metodología en los estudios migratorios y sus ejes temáticos con especial referencia a las investigaciones que abordan el contexto español y aquellas que 
han adoptado una perspectiva cuantitativa o mixta. Esta contribución realiza un rico recorrido ejemplificando estas distintas aproximaciones y ejes temáticos en donde destacan los estudios sobre la integración relacional, los análisis sobre apoyo social, transnacionalismo y evolución de las redes. El artículo concluye con una ajustada reflexión acerca de las ventajas y desventajas del ARS. Entre las ventajas se destaca que este tipo de análisis permite ampliar la visión sobre los procesos de movilidad y de integración de la población migrante que ofrecen otras aproximaciones metodológicas en las que está ausente la información reticular. Sobre las limitaciones, el artículo señala diversos problemas relativos, entre otros, al diseño de la muestra, el carácter costoso de su aplicación, el tratamiento de los valores perdidos, así como los problemas éticos que emanan de esta metodología. El artículo concluye sosteniendo que se trata de una metodología cuyo desarrollo es todavía muy joven y que tiene amplias potencialidades no sólo dirigidas a investigaciones que se detengan en redes completas, sino que acometan su estudio longitudinal.

\section{BIBLIOGRAFÍA}

ARANGO, J. (2004).'Theories of International Migration", en International Migration and the New Millennium, Aldershot, Ashgate, pp. 15-36.

BLOCH, A. (2020): "Reflections and directions for research in refugee studies", Ethnic and Racial Studies, 43, 3, pp. 436-459.

BRETTELL, C. y HOLLIFIELD, J. (2008): Migration Theory. Talking Across Disciplines, Nueva York, Routledge.

GLICK SCHILLER, N; BASCH, L. y BLANC-SZANTON, C. (1992). Towards a Transnational Perspective on Migration: Race, Class, Ethnicity, and Nationalism Reconsidered, Nueva York, Annals of the New York Academy of Sciences.

KING, R. (2012). "Theories and Typologies of Migration: An Overview and a Primer". Willy Brandt Series of Working Papers in International Migration and Ethnic Relations3/12, Malmö, Malmö University.

PISAREVSKAYA, A; LEVY, N; SCHOLTEN, P. y JANSEN, J. (2019): “Mapping Migration Studies: An empirical analysis of the coming of age of a research field", Migration Studies, https://doi.org/10.1093/migration/mnz031

URRY, J. (2007). Mobilities, Cambridge, Polity.

VAN HEAR, N. (2010): "Theories of Migration and Social Change", Journal of Ethnic and Migration Studies, 36, 10, pp. 1531-1536. 
